EXEMPLARIa Classica

Journal of Classical Philology

19, 2015, pp. 5-27

ISSN 1699-3225

\title{
APORTACIONES A LA HISTORIA DE LA TRANSMISIÓN DE LA CONSOLATIO AD LIVIAM
}

\author{
José Antonio Bellido Díaz \\ Universidad de Huelva ${ }^{1}$ \\ jabediaz@gmail.com
}

SUMMARY

After some questions relating to an edition of Consolatio ad Liuiam, we present for the first time two new excerpts and an incunable which edits it, and restore a conjecture to its rightful author.

\section{KEYWORDS}

Consolatio ad Liuiam; Romanus biblioteca Casanatense ms. 904; Krakau, Biblioteka Jagiellonska, cod. 588; Bibliothèque de Troyes, incunables 469; Bonaccorso da Pisa.

\section{RESUMEN}

Tras algunas cuestiones relativas a una edición de la Consolatio ad Liviam, damos noticia por vez primera de dos nuevos excerpta y de una edición incunable de la misma, y restituimos una conjetura a su legítimo autor.

Palabras Clave

Consolatio ad Liviam; Romanus biblioteca Casanatense ms. 904; Krakau, Biblioteka Jagiellonska, cod. 588; Bibliothèque de Troyes, incunables 469; Bonaccorso da Pisa.

Fecha de recepción: 5/03/2015

Fecha de aceptación y versión final: 25/05/2015

I. Como primera tarea para la edición de textos clásicos, sigue siendo fundamental la colación completa (en la medida de lo posible y conveniente) de los manuscritos. Sabido es que los de la Consolatio ad Liuiam de morte Drusi son de época tardía, todos ellos copiados a partir del último tercio del siglo $\mathrm{XV}$, y además su número es relativamente bajo, dieciséis en total.

1 HUM-261 Grupo de Investigación Nicolaus Heinsius. Proyecto Consolidado FFI200801843 "OVIDIANA: edición y comentario textual de las Metamorfosis de Ovidio"(2009-2014) del MICINN. Proyecto de Excelencia HUM-4534, "Edición crítica de las Metamorfosis y Opera minora de Ovidio" (2010-2013), de la CICE de la Junta de Andalucía. Agradezco las sugerencias y correcciones de los evaluadores anónimos de la revista ExClass. 


\section{Dentro de este corpus ${ }^{2}$, M. D. Reeve $^{3}$ procede a la eliminatio codicum}

2 Habría que incluir dos mss. más, perdidos. Uno de ellos es el supuesto codex Hamerslevensis, citado por H. Oldecop, De consolatione ad Liviam, Gottingae 1911, 33-4, y F. Vollmer, Poetae Latini minores II 2, Lipsiae 1923, 16, y del que da noticia E. Lasinio, "Alcuni appunti sulla Consolatio ad Liuiam", SIFC 9, 1901, 205-6, cuando recuerda que M. Manitius, "Philologisches aus alten Bibliothekskatalogen (bis 1300)", Rhein. Mus. XLVII, 1892, 35, aporta la siguiente noticia: "Hamersleven, s. XIII (B. 56) 29 f. Ovidium de Ponto in duobus vol. 31 glossas super eundem. 32 Ovidium epistolarum. 33 glossas eiusdem. 34 Ovidium de remediis duos libros. 70 Ovidium De Licia." Manitius se pregunta cuál puede ser la obra ovidiana con el título de De Licia. ¿No habría que pensar que deba leerse De Liuia? Y, con este nombre, ¿no podría designarse la Consolatio ad Liuiam? No obstante, ya había recogido esta notica con anterioridad G. Becker en su Catalogi bibliothecarum antiqui, Bonnae 1885, 141, con la diferencia de que databa el catálogo de Hamersleven en el s. XI. El segundo manuscrito es el codex Philelphi, del que se supone que Francesco Filelfo copió, por dos veces, los vv. 319-320 en una carta fechada en 1473 (Fr. Philelphi Epistolarum familiarium libri 37, Venetiis 1502, f. 255v según R. Sabbadini, "Spigolature latine", SIFC 5, 1897, 372, n.2; en realidad la carta ocupa los ff. $255 \mathrm{v}-256 \mathrm{r}$ ). He aquí, por su interés, la transcripción de la carta (con puntuación actual y desarrollo de abreviaturas):

Franciscus Philelphus, Gabrieli Pauero. Sal.

Quaesieras a me quid sentirem de uersu illo pentametro in prima Heroidum epistula ubi Publius Ouidius Naso Penelopen fingit ita scripsisse ad Vlyssen: Hic alacer missos terruit Hector equos [= her. 1.36], at ego sum usus scilicet more meo, qui nil temere affirmare didicerim. Itaque respondi tibi cum de Rhaesi equis id intelligi nullo pacto posset, utpote qui uenisset in Troianorum auxilium, me quaesiturum ex Iliade Homeri, siquid istiusmodi offenderem. Quare cum librum quintumdecimum primo ubi et pugna Patrocli et eius interitus ab Hectore legissem, dein uero librum uigesimum secundum, et item uigesimum quartum, diligenti lectione inuestigassem, nam in his duobus libris semel et iterum tractum Hectoris cadauer ab Achille Homerus fabulatur, nihil omnino inueni quod mihi coniecturam ullam aferret siquos equos Hector aliquando terruerit. Sed cum me ad hunc ipsum Nasonem retulissem, et rem continuo et ueritatem reperi. Ita enim in consolatione de obitu Drusi Neronis ad matrem Liuiam Augustam locutus est: Hoc erat Andromachae cum uir religatus ad axes. Terruit amissos sanguinolentus equos. Ex quibus quidem uerbis duo uidelicet indubitato animaduerti, et uersum illum quinarium quo de quaesieras deprauatum esse superiorum temporum inscitia, et Nasonem nostrum non esse Homerum secutum in eam sententiam. Nam neque in Iliade neque in Vlissea quicquam istiusmodi reperias, sed alium quempiam poetam imitari uoluisse, Euripiden puto, esti nihil tamen affirmo. Extat enim Euripidis tragoedia, nomine Andromache, ubi poeta ille de tracto Hectore plura cecinit. Sed iccirco non ausim affirmare quoniam codex ille quo et ea et aliae plaeraeque tragoediae continentur non est apud me. Sed cum primum ad me redierit, rem diligentius quaeram et perscribam ad te. Caeterum quod ad illum attinet uersum, ita eum scribendi oportere non ambigo: Hic lacer amissos terruit Hector equos. Nouimus enim apud Homeros legi saeuitum esse in Hectoris interempti corpus non ab Achille solum, sed a caeteris etiam Graecis, adeo ut permultis illum uulneribus confoderint atque lacerarint. Non igitur absurde Naso secutus tragicum illum poetam Graecum, quicumque tandem is fuerit, cum uoluisset ostendere horribilem Graecorum Hectorei corporis ita cruenti immanitatem, et in ea consolatione reliquit scriptum: Hoc erat Andromachae, cum uir religatus ad axes. Terruit amissos sanguinolentus equos, et in Penelopes epistola ad durum Vlissem inquit: Illic Aeacides, illic tendebat Vlisses. Hic lacer amissos terruit Hector equos, tamquam equi etiam ipsius trahentis Achillis cum oculos in tracti iterum corporis atque lacerati conuertissent, prodigiosam deformitatem aspectu suo, dum amitterentur, expauerit. Vale Mediolani. Ex aedibus nostris, nonis februariis. M.cccclxxiii.

${ }^{3}$ M. D. Reeve, "The Tradition of Consolatio ad Liviam", RHT 6, 1976, 79-98. 
descriptorum, la mayoría de ellos como copias de ediciones, y considera que los únicos mss. válidos son $C D H L M^{4}$; en el momento en que escribió su artículo no conocía aún el manuscrito $Z$, que descubriría él mismo con posterioridad ${ }^{5}$, escrito por Iohannes Nydenna de Confluentia en $1469^{6}$, pero considera que no aporta nada a la constitutio textus. Solo Schoonhoven ${ }^{7}$ lo ha colacionado.

Además, hay que contar con los excerpta, que en una obra con tan exigua tradición textual tienen el mérito de ampliar el número de testimonios manuscritos. Hasta el momento se tenía constancia de dos códices con una pequeña antología de la Consolatio ad Liviam.

En primer lugar el códice Riccardianus 152, de la biblioteca Ricardiana de Florencia. Este códice, que lleva el título de Bartolomaei Fontii excerpta uaria. Autographus, reúne Cose notabili di vari autori latini, y debió de ser escrito en torno a $1469^{8}$. En el f. 167v contiene los siguientes versos de la Consolatio ad Liuiam, encabezados por el título Ex consolatione ouidii ad liuiam de morte drusi: 9-10, 347, 357-362, 369-374, 427-428, 443-444. Estos excerpta están precedidos en el f. 167r por el breve y famoso epigrama de Giovanni Antonio Campano, obispo de Teramo (ca. 1427-14779), como

${ }^{4}$ C ca. 1466-1468/9, Oxoniensis Bodl. Douce 146; D ca. 1466-1468/9, Dresdensis Dc 147; H 1474-1488, Harvardianus Latinus 42 F. (Bibl. Collegii) (olim Phillippicus 9045); L ca. 1475, Laurentianus plut. 36.2; M ca. 1466-1474, Matritensis 1482.

${ }^{5}$ Da noticia de él (Vaticanus lat. 5160) en L. D. Reynolds, Texts and Transmission, Oxford 1983,147-8. En su opinión, la mayoría de los quince manuscritos y ediciones siguientes derivan de las ediciones Romana 1471, con la obra completa de Ovidio, y Veneta 1472, con la obra de Ausonio y la Consolatio ad Liuiam.

${ }^{6}$ El texto está firmado así en f. 129v: "1469 Té̉os IO. NY." En f. 93v volvemos a encontrar

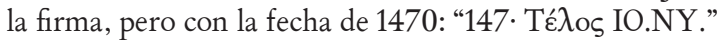

${ }^{7}$ H. Schoonhoven, The Pseudo-Ovidian Ad Liuiam de morte Drusi (Consolatio ad Liuiam, Epicedion Drusi). A critical text with introduction and commentary, Groningen 1992.

${ }^{8}$ Contiene una data no solo en el f.128r, como menciona Reeve, Collecta sub Petro Cennino Anno 1468, sino también en el f.122r, sub Bernardo B francisci anno 1467; además, en los ff. 198r-199r (el códice tiene 200 ff.) se incluye la trascripción de una carta de F. Filelfo a Nicodemo Tranquedino, respondiendo a una cuestión sobre el nombre del pez llamado lupus, fechada en 1 de julio del año 1469: Vale ex mediolano Idibus iuliis 1469. J. Amat, Consolation à Livie. Éléges à Mécène. Bucoliques d'Einsiedeln, Paris 1997, 49, se equivoca al datar este códice en el año 1463: "On y trouve les vers suivants, datés de 1463:...”. La data del f. 122r ya fue observada por Lasinio, "Alcuni appunti”, 204. Léase también a S. Caroti, S. Zamponi, Lo Scrittoio di Bartolomeo Fonzio, Milano 1974, 41-5.

${ }^{9}$ L. Moréri, Le Grand Dictionnaire historique, ou le mélange curieux de l'histoire sacrée et profane, nouvelle et derniere édition revûe, corrigée et augmentée Paris 1725, Tome II, 70. El epigrama en cuestión reza así:

Huius nympha loci : sacri custodia fontis

Dormio : dum blandae sentio murmur aquae.

Parce meum quisquis tangis caua murmura [sic] somnum

Rumpere siue bibas, siue lauere, tace.

En CIL VI.5.3.e (Epigrammata recentia, ex versibus et inscriptionibus recentibus, quae pro antiquis habitae sunt, paucas hic proposuimus) puede leerse como prefacio al epigrama: super 
indica en el título el propio Bartolomeo Fonzio (1447-1513): Romae nuper inuentum [? non liquet] A [sic] Campano episcopo factum. En el f. 168r comienza la Explanatio acronis super oratij flacci poema.

En el Monacensis Clm 7471 (Bayerische Staatsbibliothek, München), de finales del s. XV, podemos leer hasta veintiocho versos de cons.; este es el contenido del f. $2 \mathrm{r}$, donde se encuentran a dos columnas: f. 2 ra, sin indicación de título, (cons.) 369-378, Ouidius, (cons.) 427-428, Publij Ouidij nasonis de philomena liber incipit (vv. 1-44), (cons.) 13-14, (in pede) Propertius, oculi sunt in amore duces; f. 2rb: (cons.) 443-444, 357-360, 265-268, 157-

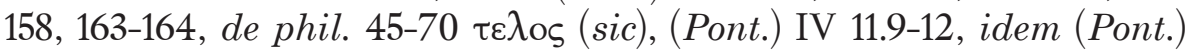
IV 8.43-48, 51; I 3.29, 35-36. En la descripción del catálogo de Halm ${ }^{10}$, no se hace ninguna alusión a este excerptum de Consolatio, por lo que tal vez pasó desapercibido a los filólogos hasta que H. Schoonhoven, haciendo unas prácticas con otros profesores y algunos alumnos de la Universidad de Groningen en la Bayerische Staatsbibliothek de Múnich, los descubrió cuando examinaba el códice ${ }^{11}$.

ripam Danuii in quo est sculpta nympha ad amoenum fontem dormiens, sub figura est hoc epigramma FERR., in viridario card. S. Clementis MAZ., in hortulo Colotiano SMET., in aedibus Caesiorum LIPS., Romae SPON., domo Vinc. Sassini in Pannaria ad Panormum CASTELL. La transcripción del epigrama recoge el correcto marmora, que Fonzio deturpó. Este epigrama había sido tomado por antiguo hasta que se le incluyó entre los falsos en este volumen del CIL. Quizá el de Fonzio sea el más antiguo testimonio del epigrama. Puede leerse a O. Kurz, "Huius nympha loci. A pseudo-classical inscription and a drawing by Dürer", Journal of The Warburg and Courtauld Institute 16, 1953, 171-7; D. Wuttke, "Zu huius nympha loci", Arcadia 3.3, 1968, 306-7; E. B. MacDougall, "The Sleeping Nymph: Origins of a Humanist Fountain Type”, The Art Bulletin, Sep. 1, 1975, 357-65.

${ }^{10} \mathrm{~K}$. Halm, G. von Laubmann, W. Meyer, Catalogus codicum manu scriptorum Bibliothecae Regiae Monacensis. Tomi III. pars III. codices Latinos (Clm) 5251-8100 complectens, Monachii A. M.D.CCC.LXXIII, Unveränderter Nachdruck Weisbaden 1968, 163. Contiene la siguiente descripción: 7471 (Indersd. 71) 2º. s. XV-XVI. 149 fol. f. 1 sq. Ovidii Philumena liber. Catullus ad Ciceronem. Ex Tibullo et Martiale excerpta. f. 3 M. Tulli Ciceronis ad Quintum fr. de oratore liber I. f. 42 Crispi Salustii liber de coniuratione Catilinae. Adiecta sunt f. 62v epitaphia Dymii (Hues, bone quis? Dymus, etc), cynici cuiusdam philosophi (Condor ego hic cynicus, etc) et epigramma Ausonii de cynico Diogene. f. 63 Catilinae oratio responsiva in M. Tullium Ciceronem. Inc. Omnes homines qui in maximis principatibus uitam agunt etc. f. 65 L. Florii (sic) epitomatis liber IIII de bello Catilinae. f. 65 De laude Julii Caesaris (ex Plinii N.H. VII, c. 25). Sequuntur laudes Pompei Magni, Catonis primi (sic), diversae multorum. f. 66 P. Virgilii Maronis Moretum, f. 68 Rosetum (cf. Meyeri Anthol. n. 1023). f. 69 Eiusdem liber Priapeiorum. f. 79 Copa. f. 80 Anei Senecae Corduvensis Hercules furens. Sequuntur octo disticha, quorum initium: Piramides nili. pharos insula. Juppiter hammon etc. f. 101 P. Ovidii Nasonis opusculum de pulice. Inc. Parva pulex et amara lues inimica puella etc. Sequuntur versus ex eius epist. ex Ponto II, 5, 59 sqq. Scilicet ingeniis aliqua et concordia iunctis etc. Versus Petronii $=$ Meyeri Anthol. n. 160. f. 102 Q. Horatii Flacci sermonum lib. I et II, 1. f. 122 Libri de arte poetica v. 1-270. f. 132 C. Plinii Secundi nouocomensis ad T. Vespasianum praefatio in libris naturalis historiae. f. 135 Dictionum elegantium interpretatio lecta ad elegantias Laurentii Vallae. f. 136 Deorum et heroum antiquorum explicatio.

${ }^{11} \mathrm{H}$. Schoonhoven, "Another Excerpt from the Consolatio ad Liviam", Mnemosyne $4: 37: 1 / 2,1984,147-8$. 
Pues bien, en nuestra labor de recopilación e investigación de testimonios manuscritos de esta obrita hemos redescubierto dos desconocidos hasta ahora. El primero es el ms. Romanus biblioteca Casanatense ms. 904, Florilegium sententiarum ex latinis scriptoribus excerptarum ${ }^{12}$, del s.

12 W. Gardner, "The Manuscripts of Catullus", CPh 3:3, 1908, 233-56 (en p. 241). $\mathrm{La}$ descripción completa se encuentra en http://manus.iccu.sbn.it//opac SchedaScheda. php?ID=16013 (consultado por última vez el 25 de mayo del 2015): 2r-3v De gestis Alexandri (Q. Curtius), 6r-9r Lactantius Firmianus, 9v-13r Persius, 13r-15v Silius Italicus, 17v-24v Quintilianus, 25r-29v (Martialis), 30r-36v de legibus (Plato), 36r-36v in epistolis (Plato), $36 \mathrm{v}$ in libro de philosophia; in libro de uirtute (Plato), 37r de uoto (Plato), 37v-38r de regno (Plato), 38r Cratilus (Plato), 38v-39r conuiuium (Plato), 39r Phaedrus, Apologia, Phedon (Plato), 39v-40r de republica (Plato), 40r homo (Plato), 40v-42r de natura deorum (Cicero), $42 \mathrm{r}$ de diuinatione (Cicero), 42r-43v in tusculanis (Cicero), 43v-44r de finibus bonorum et malorum (Cicero), 44r de esentia mundi siue Timeo Platonis (Cicero), 44r-46r de legibus (Cicero), 46r-46v in Verrem accusationum (Cicero), 46v-47r in Philippicis (Cicero), $47 \mathrm{r}-51 \mathrm{r}$ de amicitia (Cicero), $51 \mathrm{r}-52 \mathrm{v}$ de senectute (Cicero), $52 \mathrm{v}-53 \mathrm{v}$ ex paradoxis (Cicero), $53 \mathrm{v}-54 \mathrm{v}$ in rethorica noua (Cicero), $54 \mathrm{v}-55 \mathrm{v}$ in rethorica ueteris (Cicero), $55 \mathrm{v}$ Cicero de oratore, 55v-57v Cicero ad Brutum, 57v-61v Tullius Cicero de officiis libro primo, 61v-62v Cicero in familiaribus, 63r-66r de naturalis historia (Plinius), 66r-70r Caii Plinii Secundi in epistolis, 70r-70v de architectura ad Caesarem (Vitrubius), $70 \mathrm{v}-72 \mathrm{v}$ de re militari (Vegetius), $72 \mathrm{v}$ de bello iudaico (Iosephus), $73 \mathrm{r}-75 \mathrm{v}$ in epistolis (Phallarius), 75v-76v in andria (Terentius), 76r Terentius, 76v Eunuchus (Terentius), 77r Ecyra (Terentius), 77r-v Phormio (Terentius), 77v Amphitrio (Plautus), 77v-78r Asinaria (Plautus), 78r Aulularia, Captiui duo, Gurgulio (Plautus), 78r-v Cystellaria (Plautus), 78v Epidicus, Bachides, Dimitrade, Mustellaria, Manechmi, Sarsinate malis (Plautus), $79 \mathrm{v}-84 \mathrm{r}$ in georgicis (Hesiodus), 84r-v in theogonia (Hesiodus), $84 \mathrm{v}$ in bucolicis (Teocritus), $84 \mathrm{v}$ de inuidia et odio (Plutarchus), 85r-89r de remedio utriusque fortune (Plutarchus), 90rv Tibullus, 91v-95r Propertius, 95r (Catullus), 95r-97r de raptu Prosèrpine (Claudianus), 97v-104v (Iuuenalis), 104v-105v (Martialis), 105v-106r ad Martialem amicitia (Martialis), 106r-110v Lucanus, 11r-114v Metamorphoseos (Ouidius), 114v-116v in Elegiis (Ouidius), 116v-119r De ars amandi (Ouidius) [sic], 119r-121r de remedio amoris (Ouidius), 121r-125r de tristibus (Ouidius), 125v-128r de Ponto (Ouidius), 128r-v de consolatione ad Liuiam Augustam (Ouidius), 128v-129r in epistolis (Ouidius), 129r-130v de medicamine faciei (Ouidius), 130r-131r in bucolicis (Vergilius), 131r-v in georgicis (Vergilius), 133r-133r Aeneados (Vergilius), 133r-135r de livore (Vergilius), $134 \mathrm{r}$ in elegia obitus Moecenatis (Vergilius), 135r-141r in odis (Horatius), 141r Epodon (Horatius), 141r-142v de arte poetica (Horatius), $142 \mathrm{v}-145 \mathrm{v}$ Satira primo Oratii, $145 \mathrm{v}-147 \mathrm{v}$ in epistolis liber primus (Horatius), 148r-149r de fastis (Horatius) [??], 149r de tristibus (Horatius) [??], 150r-166v in tragedis (Seneca), 166v-169v Hercules oetus (Seneca), 169v-199r in epistolis ad Lucillium (Seneca), 199r-200v de clementia (Seneca), 200v-205v de beneficiis liber primo (Seneca), 205v-208v de diuina prouidentia (Seneca), 208v-210v de uita beata ad Callionem (Seneca), 210v-215v de consolatione ad Marciam (Seneca), 215v-218r de tranquillitate vite ad serenum (Seneca), $218 \mathrm{v}-22 \mathrm{v}$ de breuitate uite ad Paulinum (Seneca), 22v-223r de studiis liberalibus (Seneca), 223r-227r de naturalibus quaestionibus ad Lucilium (Seneca), 228v de declamationibus (Seneca), 228v de remediis fortuitorum ad Gallione (Seneca), 228v-242r? de moribus (Seneca), 242r-242v incipit epistula Senece ad Paulum, 242v Annaeus Seneca Paulo Theophilo salute, 244r-v, Phisicorum (Pseudo Aristoteles), 244v-245r de celo et mundo (Pseudo Aristoteles), 245r de generatione et corruptione (Aristoteles?), 245r Temisti, 245r de memoria et reminiscentia, $245 \mathrm{r}$ de somno et uigilia, 245r-247v Ethicorum primus (Pseudo Aristoteles), 247v-248r de bona fortuna (Pseudo Aristoteles), 248r Ichonomicorum 
$\mathrm{XV}$, sin que se pueda precisar más; en sus 246 folios recopila distintos pasajes de autores latinos, y en los ff. 111r-130v se encuentran los de Ovidio ${ }^{13}$ : ff. 111r-114v Metamorphoseos, 114v-116v In Elegiis, 116v-119r De ars amandi (sic), 119r-121r De remedio amoris, ff. 121r-125r De tristibus, 125v-128r De Ponto, 128r-v De consolatione ad Liviam Augustam, 128v-129r In epistolis, 129r-130v de medicamine faciei. El ordenamiento de estos excerpta de la obra ovidiana tiene el interés de que encuadra la Consolatio entre las Epistulae ex Ponto y las Epistulae heroidum. He aquí lo que contiene el folio 128: f. 128r, (Pont.) III 7.27-28, 2.103-104, 3.101-102, 4.2122; de consolatione ad liuiam Augustam 357-360, 369-374, 427-428, 443447; f. 128v, (cons.) 448-450; in epistolis 15.31-32, 2.62, 85-86, 4.89-90, 5.7-8, 103-104, 144, 7.130, 9.29-32, 16.7-8, 344, 17.98, 130 y (met.) 1.392.

El otro nuevo excerptum de Consolatio se encuentra en el códice Krakau, Biblioteka Jagiellonska, cod. 588, ca. 1520-1530, chartaceus. En los ff. 290v-291r se encuentran los versos siguientes, encabezados por el título ad liuiam: 13-14, 17-18, 47-48, 61-62, 93-94, 97, 209-210, 213-214, 234235, 265-266, 357-362, 369-374, 443-444, 447-450 y 473-474. Resulta interesante el hecho de que los ff. 1r-v y 292r-v de este códice contienen excerpta de Ovidio, siendo los del primer folio de las Epistulae ex Ponto (I 3.17-24, 5.31-32, 6.7-8 (bis), 9.39-40, 10.17, 30; II 2.17-18, 3.7-14, 19-20, 23-24, 35-36, 5.21-22, 9.47-48, III 1.39-40, 2.9-10, 31-32, 4.7-8, 70-80, 5.12, 17-18, 6.35-36, 7.25-28, 9.17-18, 47-48, IV 3.35-36, 41-50, 57-58, 8.49-50, 10.5-6, 9-10, 11.11-12, 13.41-42, 14.31-38, 16.1-26, 49-50) y las del último de Consolatio y de Epistulae heroidum (4.89-92, 133-134, y 5.7-8).

Excepto el de Fonzio, los demás testimonios encuadran los versos de consolatio entre otros de otras obras de Ovidio: con de Philomela y Pont., el Monacensis, y entre Pont. y epist. los otros dos. Sin embargo, el hecho de que no contengan los mismos versos de las otras obras ovidianas no permite concluir que existiera una fuente común para los de consolatio.

En el siguiente cuadro comparativo de los cuatro excerpta puede comprobarse las coincidencias de versos en la consolatio (en negrita).

(Pseudo Aristoteles), 248r-249v Politicorum (Pseudo Aristoteles), 249v-250r Retoricorum (Pseudo Aristoteles), 250r in epistolis ad Alexandrum (Pseudo Aristoteles), 250r-v de regimine principum (Pseudo Aristoteles), 250v de pomo et morte (Pseudo Aristoteles), 251r de predicamentis (Pseudo Aristoteles), 251r Priorum (Gilbertus Puretanus), 251r-v Topicorum (Gilbertus Puretanus), 251v de animalibus (Gilbertus Puretanus), 251v-264r De consolatione philosophiae (Boethius), 264r-v de uita scholastica (Boethius), 264v-265v De rerum natura (Lucretius), 265v de deo Socratis (Apuleius).

${ }^{13}$ J. A. Estévez Sola, "New manuscript witnesses of Ovid's Metamorphoses”, ExClass 17, 2013, 189-206, número 39. 


\begin{tabular}{|c|c|c|c|}
\hline $\begin{array}{c}f \\
\text { Riccardianus } 152 \\
\text { ex consolatione } \\
\text { ouidij ad liuiam } \\
\text { de morte drusi }\end{array}$ & $\begin{array}{c}m \\
\text { Monacensis Clm } \\
7471 \\
\text { Ouidius } \\
\text { (el orden de versos } \\
\text { es: } 369-378,427- \\
428,13-14,443- \\
444,357-360, \\
265-268,157-158, \\
163-164 \text { ) }\end{array}$ & $\begin{array}{c}r \\
\text { Romanus } \\
\text { Casanatensis } \\
\text { ms. 904 } \\
\text { de consolatione } \\
\text { ad liuiam } \\
\text { Augustam }\end{array}$ & $\begin{array}{c}c \\
\text { Cracoviensis Bibl. } \\
\text { Jagellonicae cod. } \\
588 \\
\text { ad liuiam }\end{array}$ \\
\hline \multicolumn{4}{|l|}{$9-10$} \\
\hline & 13-14 & & $13-14$ \\
\hline & & & $17-18$ \\
\hline & & & $47-48$ \\
\hline & & & $61-62$ \\
\hline & & & $93-94$ \\
\hline & & & 97 \\
\hline & $157-158$ & & \\
\hline & $163-164$ & & \\
\hline & & & $209-210$ \\
\hline & & & $213-214$ \\
\hline & & & $234-235$ \\
\hline & $265-266,267-268$ & & $265-266$ \\
\hline \multicolumn{4}{|l|}{347} \\
\hline $357-360,361-362$ & $357-360$ & $357-360$ & $357-360,361-362$ \\
\hline $369-374$ & 369-374, 375-378 & $369-374$ & $369-374$ \\
\hline $427-428$ & $427-428$ & $427-428$ & \\
\hline \multirow[t]{2}{*}{$443-444$} & $443-444$ & $\begin{array}{l}443-444,445-446 \\
447-450\end{array}$ & $443-444,447-450$ \\
\hline & & & $473-474$ \\
\hline 19 (3 exclusivos) & 28 (10 exclusivos) & 20 (2 exclusivos) & 39 (17 exclusivos) \\
\hline \multicolumn{3}{|c|}{14 versos en común } & \\
\hline \multicolumn{4}{|c|}{12 versos en común } \\
\hline & entre los cuatro & ecen 56 versos & \\
\hline
\end{tabular}


De la comparación pueden extraerse los siguientes datos:

1. Todos ellos presentan algún verso que no se encuentra en los otros: $f$, tres $(9-10,347) ; m$, diez $(157-158,163-164,267-268,375-378) ; r$, solo dos (445-446); c, diecisiete (17-18, 47-48, 61-62, 93-94, 97, 209-210, 213-214, 234-235, 473-474), siendo el único que recoge el dístico final del poema.

2. Todos ellos coinciden en doce versos: 357-360, 369-374 y 443-444. Se trata de versos sentenciosos, que, reforzando el tema particular de la muerte de Druso, reflejan el motivo de la ineluctabilidad de la muerte.

3 . En dos versos, 427-428, coinciden tres de ellos, $m f r$. de $c$ :

4. Coinciden por parejas en los siguientes versos, siempre con presencia

$m c:$ 13-14, 265-266

$f c: 361-362$

$r c: 447-450$

Aun sin descartarlo, no creemos que haya motivos suficientes para considerar que estos excerpta provengan de un florilegio anterior, como se ha insinuado cuando solo se conocían dos de ellos, $f$ y $\mathrm{m}^{14}$. Puestos a elucubrar, en ese supuesto florilegio anterior deberían constar al menos los 56 versos totales que ofrecen $f m r c$. El hecho de que $f m r$ coincidan en catorce versos, dos más (vv. 427-428) que si incluyéramos a $c$, podría dar pie a pensar, incluso, en un segundo florilegio del que derivarían estos, mientras que $c$ quedaría solo en otra línea de la tradición, con un esquema como el siguiente:

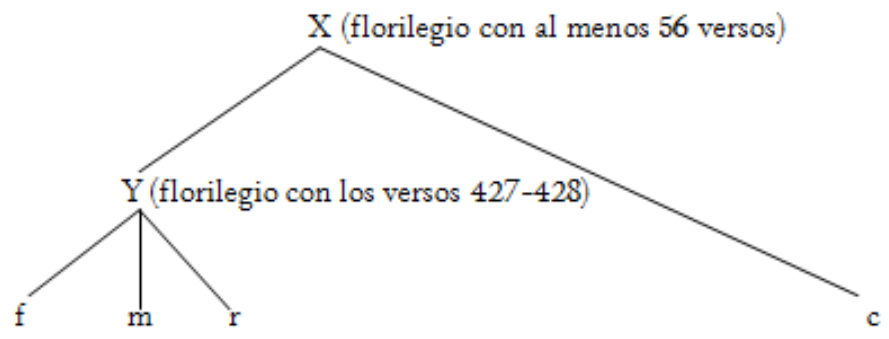

De la comparación entre $f, m$ y $r$ no se obtienen datos estemáticos o de filiación concluyentes. Veamos unos cuantos ejemplos:

357 omnes...omnes $\mathrm{fm}$ : omnis...omnis $r$

359 metam $\mathrm{frm}$ : notam $m^{s} \cdot$ unam $\mathrm{fr}$ : undam $m$

371 dispendat $f m r \cdot$ inique $f:$ ubique $m r$

372 sustinet $f r$ : sustulit $m$

427 istis $f r$ : iste $m$

444 fila $\mathrm{fm}$ : filla $r$

${ }^{14} \mathrm{~J}$. Amat, Consolation à Livie, 49, había avanzado esta hipótesis con la comparación de $f$ y $m$ : "On peut remarquer cependant que plusieurs citations coïncident, ce qui suggère un florilège antérieur." 
De ellos sacamos la conclusión de que $f r$ se enfrentan a $m$ nada menos que en tres ocasiones de un total de 14 versos en común: 359 unam / undam, en 372 sustinet / sustulit y en 427 istis / iste. Además, las lecturas de 357 omnes / omnis y 444 fila / filla oponen $f$ a $r$. Por otra parte, en 371 inique / ubique se unen $m$ y $r$ frente a $f$. Asimismo, la lectura que ofrecen los excerpta Casanatensia, $r$, en el v. 445, nebulosi in litore, los separa de una de las tres ramas de la tradición textual ${ }^{15}$, que lee nebulosum litus $(\zeta)$; en cambio, como acabamos de ver, la lectura del v. 371, ubique, coincide con la que ofrece esa misma rama. Por último, $r$ es el único testimonio de sonent frente a sonet en el v. 446 , junto con el ms. $M^{16}$. Son demasiadas divergencias para pensar seriamente en la existencia de un florilegio anterior común a todos. Hemos de añadir el hecho de que la lectura orsa, frente a ossa, que ofrece $c$ en 210, induce a pensar que, en todo caso, sería copia de la edición de Naugerius, 1516, autor de esta conjetura ${ }^{17}$, aceptada por los editores.

He aquí la colación de los cuatro excerpta, tomando como base la edición de Schoonhoven, pero con añadiduras en negrita de la de Rolán-Saquero ${ }^{18}$ adaptando sus siglas a las de aquel, cuando coincidan los mss. y edd.

$9-10(f)$

ei mihi, quam facile est (quamuis hoc contigit omnes) alterius luctu fortia uerba loqui

9 ei] hei $\Omega f \cdot$ quamuis $(-n-L \zeta) \varphi$ : quoiuis $\rho$ : cuuis $\mu$ : quantumque $M f$ : cuicumque (-cunque $C D) \psi \cdot$ omnes] omnis $\boldsymbol{M} \boldsymbol{S}^{19} \rho \boldsymbol{\mu} f^{\bullet}$

13-14 (mc)

occidit exemplum iuuenis uenerabile morum: maximus ille armis, maximus ille toga.

13 occidit] accidit $\boldsymbol{S} \cdot$ uenerabile $\Omega m c$ : memorabile $L$ : mirabile $\boldsymbol{B}^{20} \bullet$

${ }^{15}$ Seguimos a Reeve, "The Tradition", que estableció las siguientes ramas: $C=C D H ; \rho=$ $M \rho \mu$ y $\zeta=L \zeta \varepsilon$. Las letras griegas representan las ediciones: $\rho$ Romana 1471, $\mu$ Mediolanensis 1474, $\zeta$ Veneta Ausonii 1472, $\varepsilon$ Veneta 1474.

${ }^{16}$ También $U$ (Vaticanus Urb. lat. 353), que se considera una copia de una copia de $\rho$, ofrece esa lectura ante correctionem.

17 Sin embargo, A. Witlox, Consolatio ad Liviam, prolegomenis, commentario exegetico, indice instructa, Traiecti ad Mossam 1934, 84-5, atribuye erróneamente esta lectura al códice Laurentianus 36.2, $L$ (al que él asigna el siglum $A$ ), mientras que concede a Naugerius la conjetura orsa en her. 14.18, que Palmer condenó.

${ }_{18}$ T. G. Rolán, P. Saquero, Consolatio ad Liuiam de morte Drusi Neronis, Madrid 1993.

${ }^{19} S=$ Matritensis Res. 206.

${ }^{20} B=$ Londinensis Mus. Brit. Add. 11973. 
$17-18(c)$

ille genus Sueuos acre indomitosque Sicambros

contudit inque fugam barbara terga dedit,

17 Suevos] secuos $M c$ (seeuos $M$ testibus Rolán-Saquero, perperam) : saeuos ZS •

18 fugam $\Omega c$ : fugit $Z \cdot$

$47-48(c)$

nec nocuisse ulli et fortunam habuisse nocendi, nec quemquam neruos extimuisse tuos

47 nocuisse (non iusse $\boldsymbol{U}^{21}$ ) ulli (nulli $\boldsymbol{S}$ ) $\Omega c$ : nouisse nulli $\zeta$ •

61-62(c)

ille uigil, summa sacer ipse locatus in arce, res hominum ex tuto cernere dignus erat,

61 ipse] om. $c$ : ille $\boldsymbol{R}^{22}$ et Heins. $\bullet 62$ res] rex $\left.\boldsymbol{O}^{23} \boldsymbol{S U R} \cdot \operatorname{ex}\right]$ om. $\boldsymbol{O}$

93-94 (c)

lumina caerulea iam iamque natantia morte, lumina fraternas iam subitura manus.

93 iam iamque $\varphi X$ : iam tum $\psi$ : iam iam $\boldsymbol{U} c \cdot$ natantia $\chi \boldsymbol{S} c$ : nutantia $\varphi C D H \boldsymbol{B F O T} \boldsymbol{V} \boldsymbol{z}^{24}$ : mutantia $Z$ : micantia $\boldsymbol{U} \cdot$ morte $\Omega c$ : mortem $C D^{1}$.

$97(c)$

non animam apposito fugientem excepit hiatu

97 excepit $\Omega c$ : $\operatorname{traxit} \mu \cdot$

$157-158(m)$

sospite te saltem moriar, Nero, tu mea condas

lumina et excipias hanc animam ore pio.

157 mea] meas $m \cdot$

$163-164(m)$

miscebor cinerique cinis atque ossibus ossa:

hanc lucem celeri turbine Parca neat.

163 cinis] cini $\boldsymbol{V z} \cdot$ atque $\varphi$ X BFOTUVzm $: \operatorname{simul} \psi$

${ }^{21} U=$ Urbinas lat. 353.

${ }^{22} R=$ Codex Cantabrigiensis Chr. Coll. Rouse 251 .

${ }^{23} \mathrm{O}=$ Ottobonianus lat. 1469.

${ }^{24} F=$ Oxoniensis Bodl. Auct. F.I.18; T = Huntingtonensis HM 1038; V = Vat. lat. 1621; $\mathrm{z}=$ ed. Veneta 1492. 
209-210 (c)

et voce et lacrimis laudasti, Caesar, alumnum, tristia cum medius rumperet orsa dolor.

210 orsa Naugerius $c$ : ossa $\varphi$ XFFOTUVz : ora $\psi$

213-214 (c)

sed tibi debetur caelum, te fulmine pollens accipiet cupidi regia magna Iouis.

$234-235(c)$

non tibi, non ullis uincere fata datur. iste meus periit, periit arma inter et enses

234 ullis $L_{\chi} \boldsymbol{S O O U z c}:$ nullis $\boldsymbol{B F T} \xi \varepsilon \cdot 235$ periit ${ }^{1} \Omega c$ : perit $L \cdot$ periit $^{2} \Omega$ : periitque $c$ : periique $\boldsymbol{V} \boldsymbol{z} \cdot$ arma] p. arma $\boldsymbol{U}^{a c}$

$265-266(m c)$

facta ducis uiuent operosaque gloria rerum:

haec manet, haec auidos effugit una rogos.

265 operosaque $\Omega c$ : operosa $m \cdot 266$ effugit] efficit $U \cdot$

$267-268(m)$

pars erit historiae totoque legetur in aeuo

saepe opus ingeniis carminibusque dabit.

268 saepe] seque $m^{25}$ •

$347(f)$

non eadem uulgusque decent el lumina rerum:

347 uulgusque] uultusque $\boldsymbol{V} \cdot$ decent] petunt $\psi \cdot$ lumina rerum] limina regum $M \mu f$ : culmina rerum Scal. $•$

$357-360(\mathrm{fmrc})$

fata manent omnes, omnes expectat auarus

portitor et turbae vix satis una ratis.

tendimus huc omnes, metam properamus ad unam, omnia sub leges mors uocat atra suas.

$\mathbf{3 5 7}$ omnis omnis $\boldsymbol{\Omega} r$ : omnes omnis $\mathbf{Z H m}$ : omnes omnes $\boldsymbol{M L} \boldsymbol{\mu} f \boldsymbol{c} \cdot \mathbf{3 5 8}$ est turbae $Z H \cdot 359$ metam] notam $m^{s} \bullet$ unam] undam $m \cdot 360$ leges...suas] lege...sua $r \bullet$

${ }^{25}$ En la nota que Schoonhoven publicó sobre este excerptum, "Another", no dio noticia de esta variante textual y tampoco la incluyó en el aparato crítico de su edición. 
$361-362(f c)$

ecce, necem intentant caelo terraeque fretoque

casurumque triplex uaticinantur opus:

361 intentant Bentleius : intentam $\Omega f_{c} \cdot 362$ casurumque Heinsius : casurum $\Omega f_{c}$ : caussarumque $\boldsymbol{S c a l}$. : casurum unde $\boldsymbol{B a \boldsymbol { e }} \cdot \bullet^{\bullet}$ uaticinantur Heinsius : uaticinatur $\Omega f_{c} \bullet$

369-374 ( fmrc)

uita data est utenda, data est sine faenore nobis

mutua nec certa persoluenda die.

fortuna arbitriis tempus dispensat iniquis:

illa rapit iuuenes, sustinet illa senes,

quaque ruit, furibunda ruit totumque per orbem

fulminat et caecis caeca triumphat equis.

369 nobis $\Omega$ fmrc : non bis $C D H^{s} \bullet 371$ dispensat] dispendat $m \bullet$ iniquis $Z H$ : inique $\chi C D S f$ : ubique $\varphi$ BOUVzmrc $\mathbf{3} 72$ sustinet $\chi \psi \boldsymbol{S} f r$ : sustulit $\varphi$ FOTUVzmc : substulit $\boldsymbol{B} \bullet$

$375-378(m)$

regna deae immitis parce irritare querendo,

sollicitare animos parce potentis erae.

quae tamen hoc uno tristis tibi tempore uenit,

saepe eadem rebus fauit amica tuis.

376 erae] herae $\Omega m$ •

$427-428(\mathrm{fmr})$

supprime iam lacrimas: non est reuocabilis istis,

quem semel umbrifera nauita lintre tulit.

$\mathbf{4 2 7}$ istis $\Omega$ fr : iste $m \cdot \mathbf{4 2 8}$ lintre] lintra $Z$ •

$443-444$ ( $f m r c)$

sed rigidum ius est et ineuitabile mortis,

stant rata non ulla fila tenenda manu.

443 est ineuitabile $Z^{26} \bullet \mathbf{4 4 4}$ stant nonulla rata $Z \cdot$ fila] ulla $r^{a c} \bullet$

$445-446(r)$

ipse tibi emissus nebulosum in litus Auerni,

si liceat, forti uerba tot ore sonet.

445 nebulosum in litus $S$ choonhoven : nebulosum ad litus $\boldsymbol{V} \boldsymbol{z}:$ nebulosum lit(t)us $\varphi$ : nebulosi litore Heins. : nebulosi in lit(t)ore $\chi \psi S$ : nebulosi in litori $r \cdot 446$ si $V$

Burman : sic $\Omega r$ : liceat] licet $V \cdot$ sonent $M U r \bullet$

${ }^{26}$ Hubiera sido preferible "et om. $Z$ ". 
$447-450(r c)$

"quid numeras annos? vixi maturior annis:

acta senem faciunt, haec numeranda tibi,

his euum fuit implendum, non segnibus annis:

hostibus eueniat longa senecta meis.

448-449 om. $U \cdot \mathbf{4 5 0}$ meis Scaliger : metu $\Omega$ rc : situ $\boldsymbol{B}^{2}$

$473-474(c)$

est coniunx, tutela hominum, quo sospite uestram,

Liuia, funestam dedecet esse domum.

Por lo que respecta a $c$, presenta errores en 17 Suevos] secuos $M c$ (seeuos $M$ testibus Rolán-Saquero, perperam) : saeuos ZS, y en 61 ipse] om. c : ille $\boldsymbol{R}$ et Heins. Se acerca a $U$ en 93 iam iamque $\varphi X$ : iam tum $\psi$ : iam iam $\boldsymbol{U} c$, pero se separa de él en el mismo verso 93 natantia $\chi \boldsymbol{S} c$ : nutantia $\varphi$ CDHBFOTV $\boldsymbol{z}^{27}$ : mutantia $Z$ : micantia $\boldsymbol{U}$. El dato más concluyente sobre $c$ se observa en 210 orsa $c$ Naugerius : ossa $\varphi X \boldsymbol{B F O T U V \boldsymbol { z }}$ : ora $\psi$, que induce a pensar, como decíamos anteriormente, que sería copia de la ed. de 1516 del humanista italiano. Sin embargo, esto no es una prueba definitiva, pues se separa de la Aldina II. en no pocos pasajes: 17 secuos frente a Sueuos de Naugerius, 61 ipse omitido por $c, 93$ iam iam frente a iam iamque de Naugerius. Dentro de los testimonios manuscritos, ofrece una lectura única en 235 periit $^{2} \Omega$ : periitque $c$ : periique $\boldsymbol{V} \boldsymbol{z} \cdot$ arma] p. arma $\boldsymbol{U}^{a c}$, que habría que interpretar como un intento por parte del autor del florilegio de regularizar el hexámetro iste meus periit, periit arma inter et enses; sorprendentemente, Naugerius también editó periit periitque ${ }^{28}$; ese mismo intento de regularización ha provocado las anómalas lecturas de $V z$ y de $U$. También coincide con la lectura de Naugerius en sustulit de $\mathbf{3 7 2}$ sustinet $\chi \psi \mathbf{S} f r$ : sustulit $\varphi$ FOTUVzmc : substulit $\boldsymbol{B}$. Ante esta situación de discordancias y coincidencias, cabría preguntarse si el autor del compendio $c$ se basó en alguno de esos manuscritos antiguos que Navaggero dice haber colacionado para su edición y que no se haya conservado ${ }^{29}$.

${ }^{27} F=$ Oxoniensis Bodl. Auct. F.I.18; T = Huntingtonensis HM 1038; V = Vat. lat. 1621; $\mathrm{z}=$ ed. Veneta 1492.

${ }^{28}$ No obstante, Willox, Consolatio ad Liviam, 93, avisa de que en los compuestos de ire se alarga con mucha frecuencia la sílaba final en Plauto y en Ovidio.

${ }^{29}$ En efecto, en el prefacio al segundo volumen de la edición Aldina de la obra completa de Ovidio introdujo Naugerius sus anotaciones críticas, y en las páginas 44-45 (que están sin numerar) se encuentran las de la Consolatio. Para refrendar algunas de las lecturas que propone, se basa en la lectura de antiguos códices con expresiones como in ueteribus rectius o ex ueteribus legendum. Pueden leerse estas anotaciones también en Andreae Naugerii patrici Veneti oratoris et poetae clarissimi opera omnia, Patavii 1718, 166-7 (con numeración de los versos). Dado que en estas notas críticas no menciona Naugerius el v. 210, en el que edita orsa, 
Por su parte, $r$ comete un error exclusivo en $\mathbf{3 6 0}$ leges...suas] lege...sua $r$, mientras que se aparta de los otros excerpta al ofrecer el ac. pl. en -is en $\mathbf{3 5 7}$ omnis omnis $\Omega r$ : omnes omnis $Z \mathbf{H} m$ : omnes omnes $\boldsymbol{M L} \boldsymbol{\mu} f$.

Si bien ambos, $r$ y $c$, parecen pertenecer a la rama $L \zeta \varepsilon$ (que Schoonhoven

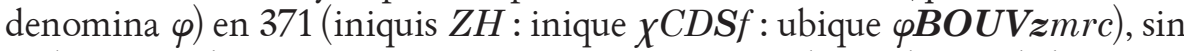
embargo en el verso siguiente se separan, manteniéndose $c$ dentro de la misma línea, mientras que $r$ sigue las líneas $\chi(M \rho \mu)$ y $\psi(C D Z H)$ : $\mathbf{3 7 2}$ sustinet $\chi \psi \mathbf{S} f r$ : sustulit $\varphi \boldsymbol{F O T U V \boldsymbol { Z } m c}$ : substulit $\boldsymbol{B}$. Parece claro, por tanto, que no debió de existir un florilegio común a los excerpta, que deben de ser producto de autores que se basan en fuentes distintas.

II. Respecto a las ediciones, hasta el momento se conocían trece incunables, que según Reeve deben organizarse así:

ค ed. Rom. 1471

$\zeta$ ed. Ven. 1472

$\varepsilon$ ed. Ven. 1474: fuente para Parm. 1477, Mediol. 1477, Vic. 1480, Bonon. 1480, Ven. 1486, Ven. 1492

$\mu$ ed. Mediol. 1474: fuente para Mediol. 1478, una edición sine loco et anno y Mediol. 1486

Dado el espectacular avance de las nuevas tecnologías, distintas bibliotecas de diversos países están poniendo a disposición del público a través de internet gran parte de sus fondos bibliográficos en formato digital. Esto nos está permitiendo consultar muchos fondos a los que de otra manera nos habría sido muy difícil, si no imposible, acceder, hasta el punto de que hemos detectado, como ya se ha comentado al principio, muchos testimonios manuscritos de la obra ovidiana no tenidos en cuenta hasta ahora. Pues bien, podemos anunciar que hemos encontrado en la Bibliothèque de Troyes un ejemplar de una edición s.l., s.a. desconocida hasta ahora de la Consolatio ad Liuiam, pero que es lo más probable que se imprimiera en París en torno al año $1480^{30}$ (la denominaremos $\pi$ ). La importancia de esta edición estriba en que es la primera de la que tengamos constancia que se imprimió fuera de Italia. Se conserva en un misceláneo de varias obras de ediciones incunables reunidas en este solo

puede suponerse que se trata de una conjetura suya.

${ }^{30}$ Dado que la URL es excesivamente larga, preferimos que el lector compruebe el dato haciendo la búsqueda "consolatio ad liviam" por sí mismo en http://www.mediatheque.grandtroyes.fr/cat/Vubis.csp?Profile=Default\&SearchMethod=Find_1. En el último intento de acceder al documento, el 25 de mayo del 2015, no se encontraba disponible por problemas técnicos. 
volumen. Está compuesto de diez unidades codicológicas ${ }^{31}$, y corresponde a nuestro poema la última de ellas, con el número de catálogo 469:ff. $265 \mathrm{r}-277 \mathrm{v}^{32}$. Publii Ouidii Nasonis Poetae consolatio ad Liuiam augustam de morte Drusi Neronis filij eius qui in Germania morbo periit, Paris?, 1480?. Bibl.: Jean-Marie Arnould, Catalogues régionaux des incunables, t. I, 1132. Bien es verdad que no aporta prácticamente nada a la constitutio textus de la Consolatio, pero aun así vamos a hacer una comparación de las lecturas de $\pi$ con las que le sirvieron a M. D. Reeve ${ }^{33}$ para establecer las tres ramas de la tradición de esta obrita y así encuadrarla en alguna de ellas.

${ }^{31}$ Estas son las nueve primeras unidades codicológicas (460-468):

- 460: ff. 1r-13v: Stella clericorum, Paris, ed. Pierre Levet, ca. 1489. Bibl.: Jean-Marie Arnould, Catalogues régionaux des incunables, t. I, 1361; British Museum, Catalogue of books printed in the XVth cent., VIII, 104.

- 461: ff. 14r-19v: Regulae grammaticales, Paris, ed. Pierre Levet, ca. 1488. Bibl.: JeanMarie Arnould, Catalogues régionaux des incunables, t. I, 1254.

- 462: ff. 20r-86v: Expositio hymnorum, Paris, ed. Pierre Levet, 8 dic. 1488. Bibl.: JeanMarie Arnould, Catalogues régionaux des incunables, t. I, 590; Ludwig Hain, Repertorium bibliographicum, 6782.

- Dos ff. en blanco sin numerar.

- 463: ff. 87r-99v: Jean Vivet (Johannes Vivetus), Tractatus de orthographia, Rouen, ed. Guillaume Le Talleur, ca. 1487. Bibl.: Jean-Marie Arnould, Catalogues régionaux des incunables, t. I, 1511.

- 464: ff. 100r-136v: Robert Gaguin (Robertus Gaguinus) (1434?-1501), Ars uersificatoria, Paris, ed. Pierre Levet, 1492-1500. Bibl.: Jean-Marie Arnould, Catalogues régionaux des incunables, t. I, 632.

- 465: ff. 137r-214v: Johannes (Johannes Sulpitius) (1430?-post 1490), De arte grammatica siue de octo partibus orationis, Paris, ed. ?, ca. 1500. Bibl.: Jean-Marie Arnould, Catalogues régionaux des incunables, t. I, 1373.

- 466: ff. 251r-244v: De mundissimo Virginis Mariae conceptu, Robert Gaguin (Robertus Gaguinus) (1434?-1501); Fernand, Charles (Carolus Fernandus) (1450?-1496?) [comment.], Paris, ed. Antoine Vérard?, 28 mayo 1489 (in fine "Parisii MCCCCnono"). Bibl.: Jean-Marie Arnould, Catalogues régionaux des incunables, t. I, 637; W. A. Copinger, Supplement to Hain's Repertorium bibliographicum, I, 7415; British Museum, Catalogue of books printed in the XVth cent., VIII, 96.

- 467: ff. 245r-251r: De praeparatione ad missam, Bonaventure (Bonaventura) (1221?1274);

ff. 251r-252v: Meditatio de ss. sacramento altaris, Jean Pecham (Johannes Pechamus) (1220?-1292), Paris, ed. Pierre Levet, 1486-90. Bibl.: Jean-Marie Arnould, Catalogues régionaux des incunables, t. I, 336; W. A. Copinger, Supplement to Hain's Repertorium bibliographicum, II, 3544; Gesamtkatalog der Wiegendrucke, 4669.

- 468: ff. 253r-264v: Cicero, Paradoxa stoicorum, Paris, ed. Philippe Pigouchet, ca. 1490. Bibl.: Jean-Marie Arnould, Catalogues régionaux des incunables, t. I, 462; British Museum, Catalogue of books printed in the XVth cent., VIII, 112; Gesamtkatalog der Wiegendrucke, 7015.

${ }^{32}$ Hay un salto de paginación desde el folio 267 al 269 por error, sin que esto afecte al texto, que pasa del verso 118 en el f. 267v al verso 119 en el f. 269r.

${ }_{33}$ M. D. Reeve, "The tradition", RHT 6, 1976, 79-98. Una revisión profunda de esta propuesta, con nuevas sugerencias, puede leerse en Rolán-Saquero, Consolatio ad Liuiam, 43-57. 
1. Respecto a las interpolaciones que definen la rama formada por $C D H$, nuestra edición se mantiene siempre al margen:

95 sumpsit $C D H$ : fregit vel fiegit vel figit cett. (figit $\pi$ )

103 etiam derisa malignos $C D$ (delusa $H Z$ ) : malos irrisaque tales cett. $\pi$ 125 qui...eras $C D H(Z)$ : heu...ubi es cett. $\pi$

163 simul $C D H(Z)$ : atque cett. $\pi$ (adque)

404 divini $C D H$ : et immensi cett. $\pi$

2. Lo mismo sucede con las innovaciones de la rama $M \rho \mu$ :

76 fati $M \rho \mu$ : fati haec cett. $\pi$

116 gravibus $M \rho \mu$ : gravidis cett. $\pi$

321 furibunda $M \rho \mu$ : ferienda cett. $\pi$

356 quin tu $M \rho \mu$ : quam si cett. $\pi$

366 ex qua natus $M \rho \mu$ : et qua natus $L \zeta \varepsilon \pi$ [quantus $C D H(Z)$ ]

402 tua $M \rho \mu$ : tria cett. $\pi$

3. Según se deduce, $\pi$ está estrechamente unida a la rama $L \zeta \varepsilon$, y así lo demuestran las siguientes lecturas $(\mathrm{C}=\mathrm{CDH} ; \rho=\mathrm{M} \rho \mu ; \zeta=\mathrm{L} \zeta \varepsilon)$ :

3 miserabile $C \rho$ : mirabile $\zeta \pi$

9 quamvis $\zeta \pi$ : quoivis $\rho$ : cuicunque $C$ : cuuis $\mu$ : quantumque $M$

34 os oculos $\rho$ : osque oculos $\xi H \pi$ : os oculosque $C$

75 incassum $\xi H \pi$ : in longum $C D \rho$

78 magni $\zeta \pi$ : -um $C \rho$

$79^{34}$ turba $C \rho \pi^{2}$ : verba $\zeta \pi$

101 zephyris $C \rho$ : -us $\zeta \pi$

111 Clymeneides $C \rho \pi^{2}:$-dos $\zeta \pi$

131 non ego $C \rho$ : ego non $\zeta \pi$

141 quos $C \rho:$ quo $\zeta \pi$

149 referetur $C \rho:$-atur $\zeta \pi$

172 patriae $C \rho$ : prime $\zeta \pi$

215 quod $C \rho$ : quid $\zeta \pi$

240 pollice....pensa severa $\zeta \pi$ : impia...pollice pensa $C \rho$

252 structaque $C \rho$ : strictaque $\zeta \pi$

304 visa $C \rho$ : iussa $\zeta \pi: o m . M \mu$

307 per $C \rho: \operatorname{pro} \zeta \pi$

349 alto $\zeta \pi$ : alte $C \rho$

354 quod $C \rho \mu$ : quo $\zeta M \pi$

371 iniquis $H$ : inique $C D \rho$ : ubique $\zeta \pi$

372 sustinet $C \rho:-$ tulit $\zeta \pi$

${ }^{34}$ Con $\pi^{2}$ (79 y 111) nos referimos a las correcciones hechas a mano en la edición por algún lector que introdujo anotaciones marginales e interlineales en este ejemplar desde el verso 75 hasta el verso 166. También intervino esa misma mano en 106 Daulias $D^{2} H^{2} B^{2} M \pi^{2}$ (Daulas) : Daunias $\Omega \pi$. 
387 rapax $C \rho: \operatorname{capax} \zeta \pi$

394 tui $C \rho$ : tibi $\zeta \pi$

395 lenissimus $C \rho:$ lev $-\zeta \pi$

396 accipere es $C \rho$ : acciperes $\zeta \pi$

403 gravis Cuperus : gravi $\zeta H \pi$ : grais $C D$ : gradus $\rho$

433 hoc $C \rho$ : haec $\zeta \pi$

445 nebulosum litus $\zeta \pi$ : nebulosi in litore $C \rho$

457 consul et $C \rho$ : consulet $\zeta \pi$

Cuando es $L$ el testimonio que se aparta del grupo, $\pi$ se mantiene con los otros dos, $\zeta_{\varepsilon}$ :

193 autem $C \rho:$ aut $\zeta \varepsilon \pi$ : haud $L$

234 ullis $C \rho L:$ nullis $\zeta \varepsilon \pi$

308 mota in $C \rho: \operatorname{motam} \zeta \varepsilon \pi:$ mota ad $L$

367 erat $C \rho L$ : erit $\zeta \varepsilon \pi$

439 nec...neque $L \rho:$ ne...neque $\zeta \varepsilon \pi$ : nec...nec $M \mu C$

Y cuando es $\zeta$ el que se aparta del grupo, $\pi$ se queda con los otros dos:

95 legit Heinsius : fregit $\rho$ : fiegit $\zeta$ : figit $L \varepsilon \pi$ : sumpsit $C$

177 consul init $C \rho$ : consulunt $\zeta$ : consuluit $L \varepsilon \pi$

Habrá que concluir, por tanto, que la edición $\pi$ se basó en la Veneta 1474, $\varepsilon$, (incluso comparte con ella las erratas 100 drusae por Druse, 248 irita por irrita, 320 teruit por terruit, y errores como 377 ibi por tibi). Ahora bien hay tres ocasiones en que $\pi$ está de acuerdo con las otras dos ramas de la tradición, $C \rho$, pero son casos en que, debido a la gran cantidad de erratas y errores que contiene la edición, se plantea la duda de si son debidos a la influencia de aquellas o a la propia edición:

84 dubitatus $C \rho \pi:$-tur $\zeta$

86 promissa $C \rho \pi$ : per- $\zeta$

104 accusatque $C \rho \pi$ : -antque $\zeta$

Además, como acabamos de indicar, esta edición no está exenta de erratas:

2 tibi $\Omega$ : tibe $\pi \cdot$ nominis $\Omega$ : nomiuis $\pi$ (también en $\mu$ )

3 Drusum $\Omega:$ drnsum $\pi$

11 fulminis $\Omega:$ ulmfinis $\pi$

26 forsitan $\Omega$ : forcitan $\pi$

54 saeuit $\Omega$ : scaeuit $\pi$

91 moriens $\Omega$ : moriems $\pi$

99 raptus $\Omega$ : raptuus $\pi \cdot$ absenti $\Omega$ : absemti $\pi$

104 annos $\Omega$ : amnos $\pi$

114 intus Heinsius : ictus $\Omega$ : ictns $\pi$ 
120 singultu $\Omega$ : singuultu $\pi \cdot$ impediente $\Omega$ : in pedinte $\pi$ 159 fratris $\Omega$ : fratis $\pi$ 162 ibis $\Omega$ : ibs $\pi$ 170 quaeque $\zeta \rho$ : queaque $\pi$ 183 clauduntque $\Omega$ : clanduntque $\pi$ 184 clamque $\Omega$ : clamqne $\pi$ 186 aspicitur $\Omega$ : apicitur $\pi$ 193 inquit $\Omega$ : inquid $\pi$ 196 Iovi $\Omega$ : ioni $\pi$ 198 preces $\Omega$ : preses $\pi$ 222 medio $\Omega$ : mepio $\pi$ 233 quiescas $\Omega$ : quiescast $\pi$ 238 opus $\Omega$ : opns $\pi$ 249 positi $\Omega$ : possiti $\pi$ 256 astra $\Omega$ : estra $\pi$ 264 viscera $\Omega$ : vicera $\pi$ 305 ultimus $\Omega$ : vultimus $\pi$ 307 moriens $\Omega$ : morens $\pi$ 308 lingua $\Omega$ : lingna $\pi$ 318 manu $\Omega$ : magnu $\pi$ 321 coruscis $\Omega$ : curuscis $\pi$ 323 amplexaque $\Omega$ : amplexeaque $\pi$ 330 inter $\Omega$ : imter $\pi$ 334 fronde $\Omega$ : frond $\pi \cdot$ vinctus $\Omega$ : vinectus $\pi$ 339 cepisse $\Omega$ : coepice $\pi$ 341 sublime $\Omega$ : sulime $\pi \cdot$ optima $\Omega$ : obtima $\pi$ 342 debuerant $\Omega$ : debueran $\pi$ 343 digna $\Omega$ : dina $\pi$ 394 oculos $\Omega$ : occulos $\pi$ 396 coacta $\Omega:$ quoacta $\pi$ 400 mollitos $\Omega$ : molitos $\pi$ 401 Iuppiter $\Omega$ : Iuppitr $\pi$ 405 feruntur $\Omega$ : furuntur $\pi$ 413 fratrisque $\Omega$ : fratisque $\pi$ 417 tamn

422 gurtura

Y tampoco de errores: 21 ignara $\Omega$ : ignora $\pi$ 24 coli est $\zeta C$ : colli est $\pi:$ coli $\rho:$ colit $M \mu$ 35 occurret $\Omega$ : occurrere $\pi$ 38 referre $\Omega$ : referro $\pi$ 51 iniuriae $\Omega$ : iniuria $\pi$ 
102 tremente $\zeta C \rho$ : tepente $M \mu$ : repente $\pi$

111 alte $\Omega$ : altae $\pi F$

115 erumpunt $\Omega$ : rumpunt $\pi$

133 inanes $($-is $\Omega)$ : in annis $\pi$

134 hos $\Omega$ : os $\pi$

141 fasces $\Omega$ : faces $\pi$

142 indiciumque $\Omega$ : indictumque $\pi$

143 matri $\Omega$ : mariti $\pi$

149 adesse $\Omega$ : adisse $\pi$

155 vereri $\Omega$ : veteri $\pi$

163 miscebor $\Omega$ : misebor $\pi$

217 more $\Omega$ : morte $\pi$

228 erat $\Omega$ : eras $\pi$

229 inhibebat $\Omega$ : inhibeat $\pi$

234 fata $\Omega$ : facta $\pi$

250 vade $\Omega$ : valde $\pi$

264 miserae $\Omega$ : miseris $\pi$

274 saeuas $\Omega$ : scenas $Z$ : sceuas $\pi$

323 maesta $\Omega$ : moestia $\pi \cdot$ natos $\Omega$ : notas $\pi$

326 tuo $\Omega$ : tua $\pi$

330 excipietur $\Omega$ : excipientur $\pi$

350 onus $\Omega$ : honus $\pi$

357 omnes (-is) $\Omega$ : omnia $\pi$

379 nata $\Omega$ : nota $\pi$

389 Dalmata $\Omega$ : Delmata $Z$ : dalinata $\pi$

396 aure $\Omega$ : auro $\pi$

397 longa $\Omega$ : longan $\pi \cdot$ luctum $\Omega$ : lucrum $\pi$

401 fati $\Omega$ : facti $Z \pi$

418 fortis $\Omega$ : forus $\pi$

440 tristia $\Omega$ : tristitia $\pi$

443 ius $\Omega$ : vis $\pi$

455 quamquam...adfuit Gronovius : quicquam...affuit $\Omega$ : quicquam...

affluit $\pi$

458 cui Lipsius : qui $\Omega$ : quid $\pi$

467 ero $\Omega$ : ore $\pi$

III. Pero, además de los manuscritos y las ediciones, hay que recuperar el cúmulo de datos que se esconden en las ediciones anotadas por los filólogos y los manuscritos con sus notas que se conservan en algunas bibliotecas. Tal sucede, por ejemplo, con los manuscritos Berolinensis Deutsche Staatsbibl. Diez. B Sant. 142a. y 142b., que guardan las anotaciones de 
Johannes Schrader a toda la obra de Ovidio ${ }^{35}$. Sus anotaciones a la Consolatio se contienen en los ff. 104r-108r del $142 a$ y en el folio 121br del 142b. En el Berolinensis Deutsche Staatsbibl. Diez. B Sant. 138 se encuentran en los ff. 58r-60r los notarum ineditarum ad Ouidium excerpta de Johann Hildebrand Withoof escritos por la mano de Hendrik Fiesemann y en los ff. 76r-83v las imitationes Ouidii excerptae ex Withofii notis adscriptis editioni Cnippingii: Ovidius Opera omnia, ed. B. Knipping, Leiden 1670, oder Amsterdam 1683, autógrafo de Johannes Schrader. En el Berolinensis Deutsche Staatsbibl. Ms. Diez. B Sant. 139, de 45 folios, están los Annotata in Albinouanum et Ouidium duplici generis, altera excerpta ex notis Heinsii et Burmanni, altera etiam emendationes continentia, autógrafo de Lodewyk Kaspar Valckenaer, y en los ff. 1r-7br se incluyen las anotaciones a la Consolatio ad Liuiam, a la pseudovidiana Nux y a las cartas de Odiseo a Penélope y de Paris a Enone de Sabino. Por último, en el Berolinensis Deutsche Staatsbibl. Ms. Diez. B Sant. $148 d$ se conservan autógrafas las Notae in Ouidii opera omnia de Nicolaus Heinsius. Las referidas a la pseudovidiana Consolatio ad Liuiam ocupan los ff. 146v-149v.

Más interés tienen otras notas de Heinsius. El insigne filólogo dejó repartidas por doquier multitud de ellas, unas más interesantes que otras, bien es verdad, en los más diversos lugares. Era costumbre suya anotar sus colaciones y conjeturas en anteriores ediciones de la obra objeto de su estudio. Así, para el caso que nos ocupa, tenemos constancia de que en la Deutsche Staatsbibliothek de Berlín se encuentran tres ejemplares de la edición de Daniel Heinsius en tres tomos de la obra de Ovidio editados en 1629 en Amsterdam y anotados todos ellos por su hijo ${ }^{36}$. Podría pensarse que todas esas anotaciones han sido tenidas en cuenta por editores posteriores, pero nuestra experiencia nos demuestra lo contrario. En efecto, no hace mucho hicimos la colación ${ }^{37}$ de las notas marginales e interlineales de N. Heinsius en la editio Aldina de 1515 de Catulo, Tibulo y Propercio, centrándonos tan solo en las de Catulo (Berol. Diez. oct. 2474). Dicho ejemplar contenía, además de las de N. Heinsius, las anotaciones

${ }^{35}$ Descripción completa en U. Winter, Die europäischen Handschriften der Bibliothek Diez. Teil 1. Die Manuscripta Dieziana B Santeniana; Teil 2. Die Libri impressi cum notis manuscriptis der Bibliotheca Dieziana, Leipzig: Zentralantiquariat der DDR, 1986, I, 115 (consultable en www.manuscripta-mediaevalia.de/).

${ }^{36}$ Son los siguientes: Bibl. Diez. quart. 1074, 1069, 1073, Bibl. Diez. quart. 1068, 1072, 1076 y Berol. Diez. quart. 1071, 1075, 1070 (nótese el desorden de las signaturas, que no corresponden al orden de los tomos). En la misma biblioteca se encuentra, asimismo, un ejemplar de la edición del propio N. Heinsius del año 1661 en Amsterdam en tres volúmenes con más colaciones y anotaciones (Berol. Diez. quart. 1077, 1078, 1079), de las que se sirvió P. Burman para su edición de la obra ovidiana del año 1727, pues Santen anotó en la guarda del primer volumen Quae insunt in hoc exemplari, suae editioni inseruisse videtur Burmannus. cf. U. Winter, I, 16-7.

${ }^{37}$ J. A. Bellido Díaz, "Las notas a Catulo de A. Petreius y N. Heinsius", ExClass 15, 2011, 123-200. 
de Antonio Petreio, fechadas en el año 1528, y alguna que otra de P. Burman el Joven. Sabido es que P. Burman reunió las muchas anotaciones dispersas de N. Heinsius a distintos autores latinos y las publicó bajo el título de Nicolai Heinsii aduersariorum libri quattuor numquam antea editi y les adjuntó sus Notae ad Catullum et Propertium nunc primum productae. Pues bien, podemos asegurar que las anotaciones contenidas en la edición Aldina superan en más del doble a las publicadas en los Adversariorum. ¿Y aportan algo estas anotaciones? Un ejemplo lo ilustrará mejor. En el reciente comentario crítico textual de Trappes-Lomax a Catulo ${ }^{38}$, este estudioso propone leer $i d$ en lugar de ei en 82.3: eripere ei noli, multo quod carius illi / est oculis seu quid carius est oculis, sin citar, pues no pudo haberlo leído en ningún aparato crítico, a N. Heinsius, que ya había defendido esa lectura con un contundente lege $\underline{i d}$ en una anotación marginal de la edición véneta, y que no había sido reproducida en los Adversariorum ${ }^{39}$.

Después de 540 años desde que apareció la primera edición de la Consolatio, a la que han seguido más de cincuenta, tres de ellas en los últimos veinte años, ¿cabe pensar que una nueva edición aportaría algo? Aunque pueda parecer lo contrario, no está todo hecho, y un ejemplo lo demostrará.

En un artículo posterior a la edición de Schoonhoven, Gautier Liberman trata, con su maestría habitual, determinados pasajes de la Consolatio ad Liuiam $^{40}$.

95-96 at miseranda parens suprema neque oscula fixit frigida nec fouit membra tremente sinu

95 fixit $B$ : fregit $M \rho$ : fiegit $\zeta$ : figit $L \varepsilon \mu$ : sumpsit $\psi$

Liberman defiende fixit frente a la conjetura de Schoonhoven ${ }^{41}$, pressit; acepta que existe el giro oscula premere con el sentido de "dar besos", a pesar de que los ejemplos que aporta Schoonhoven no son definitorios, pero considera que pressit está demasiado alejado de las variantes de que se dispone. Dado que la expresión figere oscula está bien documentada en latín (Lucr. 4.1179, Verg. Aen. 2.490, Ov. met. 4.141, Luc. 6.565, Sil. 11.331), basa su defensa en que la conjetura del manuscrito $B$ (Londinensis Mus. Brit. Add.

${ }^{38}$ J. M. Trapes-Lomax, Catullus. A Textual Reappraisal, Swansea 2007.

39 Añadamos, además, que en la misma edición aparece a pie de página una conjetura, probablemente de A. Petreio, que nos parece muy sugestiva: eripere ei noli, multo quod carius illi / est, oculos, seu quod carius est oculis.

${ }^{40} \mathrm{G}$. Liberman, "Observations sur le texte et la date de la Consolation à Livie", Mélanges de l'École française de Rome, Antiquité, 106: 2, 1994, 1119-36.

${ }^{41}$ H. Schoonhoven, The Pseudo-Ovidian:

95-96 at miseranda parens suprema neque oscula pressit

frigida nec fovit membra tremente sinu;

95 pressit scripsi : fregit $M \rho$ : fiegit $\zeta$ : figit $L \varepsilon \mu$ : sumpsit $\psi$ : legit Heinsius 
11973, que es una copia de $\varepsilon$, ed. Veneta 1474) representa el texto original, y las variantes figit, fregit, fiegit serían una deturpación de aquella. Por otra parte, considera que la conjetura de N. Heinsius, legit, aceptada por Reeve, entre otros ${ }^{42}$, introduce una expresión inédita y termina recordando que, pese a su propia conjetura, Heinsius había editado fixit.

Pues bien, resulta que el ms. $B$ no contiene la lectura fixit por ningún lado, pues lee At mis(er)anda parens sup(re)ma neq(ue) oscula fingit, y al margen, referido a sup(re)ma por medio de una llamada, escribe $u(e) l$ fracta, lectura, por otro, lado, que no cuadra métricamente y que no es mencionada en ninguna de las ediciones que hemos manejado. ¿Comete Liberman un error? Evidentemente, no, sino que se ha basado en el comentario que hace Schoonhoven ${ }^{43}$ para defender su conjetura pressit, donde recuerda que el propio Heinsius no confió lo suficiente en su propuesta legit y prefirió fixit “(the reading of $\left.B^{44}\right)$ ". Esta alusión fue la que indujo a error a Liberman. Pero lo curioso es que en su app. crit. Schoonhoven no menciona nada de ello (de él se desprende, por otra parte, que Heinsius editó legit, cuando hemos visto que no fue así).

Si pasamos ahora al app. crit. de la última editora de la Consolatio, J. Amat ${ }^{45}$, acierta en que $B$ lee fingit, pero también incluye otros errores. En primer lugar, parece indicar que fixit fue incluido en el texto por vez primera por Heinsius cuando en realidad esta lectura está documentada desde la edición de Bonus Accursius en Venecia en 1492.

${ }^{42}$ También la aceptan Rolán-Saquero, Consolatio ad Liuiam. La conjetura de Heinsius aparece en las notas a su edición de 1658, p. 498 (N. Heinsius, Publii Ovidii Nasonis operum tomus I. Scripta amatoria complexus. Nicolaus Heinsius, D. f., infinitis locis castigavit ad fidem scriptorum exemplarium., Amstelaedami, A CIO IOC LVIII): "Suprema neque oscula fixit] fregit duae editiones ex vetustioribus. Forte legit. ut de Hecuba Met. XIII. Osculaque ore legit, consuetaque pectora plangit. sic enim veteres nonnulli libri exaratum illic exhibent. sumpsit tamen hic codex unus. alli tres, figit. an fingit? ut Met. IV. de Thisbe, gelidis in vulneribus oscula fingens. sed figere oscula usitatum, nec loco movendum."

${ }^{43}$ Schoonhoven, The Pseudo-Ovidian, 111-2.

${ }^{44}$ Que se hubiera mantenido la sigla B que regía desde F. Vollmer, Poetae Latini Minores, Leipzig, 1911-1935, vol. II, fasc. 2, 1923, 15-35, para referirse al ms. Vat. Urb. lat. 353 tampoco salva la situación, pues este ms. (actualmente $U$ ) lee figit.

${ }^{45}$ Amat, Consolation à Livie:

95-96 at miseranda parens suprema neque oscula fixit frigida nec fouit membra tremente sinu;

95 fixit Heins. : figit FLOUV fingit $B$ fregit $M r$ sumpsit $C D H$ pressit $S c h o o n$. 
Los que parecen haberse acercado más a la verdad son Rolán-Saquero ${ }^{46}$, pero aún así tienen sus defectos. En efecto, el app. crit. parece indicar que Heinsius editó legit, cuando esa era su conjetura. Un simple coniecit habría deshecho el entuerto. Pero hay una anotación que podría desvelar el misterio de la aparición de fixit en la tradición textual. Según el app. crit. el ms. $V$ (Vaticanus lat. 1621, del año $1493^{47}$, que fue uno de los cuatro que manejó Heinsius) recoge la lectura fixit, aunque según J. Amat este ms. lee figit. Ante tal galimatías, no nos queda más remedio que recurrir al manuscrito, que, en efecto, da la razón a Rolán-Saquero (f. 3v):

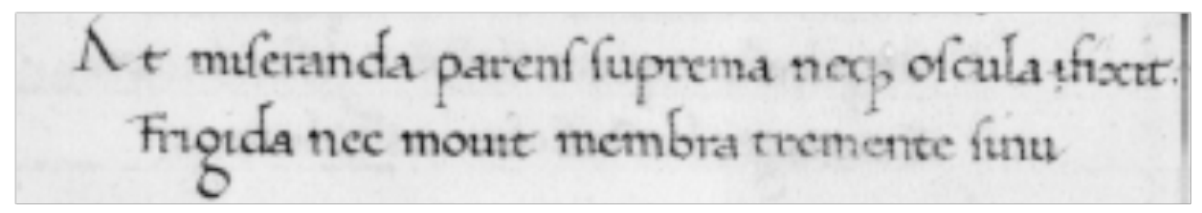

Ahora bien, ya Oldecop ${ }^{48}$ había demostrado que $V$ es una copia de la edición de Venecia de 1492, de Bonus Accursius, pues comparte con ella cinco errores significativos: 114 occulit por oculis, 125 humido por tumulo ${ }^{49}, 155$ iubet hinc por iubet hic, 235 periit periique por periit periit y 287 non sua por nec sua.

Si Oldecop, a quien sigue Reeve, está en lo cierto, cosa que no dudamos dado el aval del sabio inglés ${ }^{50}$, no queda más remedio que concluir que lo más sensato y lo más justo será restituir la paternidad de la conjetura fixit a Bonaccorso da Pisa.

${ }^{46}$ Rolán-Saquero, Consolatio ad Liuiam:

95-96 at miseranda parens suprema neque oscula legit,

frigida nec fouit membra tremente sinu;

95 legit Heinsius : figit FLOTUvzm fiegit x fingit B fregit MSr fixit ed. Ven.

(a. 1492) ifixit (cum del. sign.) V sumpsit $\mathrm{CDH}$

${ }^{47}$ En la suscriptio puede leerse: "Explicit foeliciter amen. et cetera per Alfonsum Strozam ciuem florentinum Iuuenem dignissimum. Epistola scripta fuit die XVI aprilis MCCCCLXXXXIII. Marini Tomacelli.”, en Rolán-Saquero, Consolatio ad Liuiam, 39.

${ }^{48}$ H. Oldecop, De Consolatione ad Liuiam, Diss. Gottingae, 1911, 55-73.

${ }^{49}$ Reeve corrige a Oldecop cuando indica que humido está por tumulo, no por heu modo, como decía este. Además, hemos comprobado estos errores en el app. crit. de Rolán-Saquero, el más completo de las tres últimas ediciones.

${ }^{50}$ En Reeve, "The Tradition”, 82. 
